The Astrophysical Journal, 670:557-564, 2007 November 20

(C) 2007. The American Astronomical Society. All rights reserved. Printed in U.S.A.

\title{
A RING OF WARM DUST IN THE HD 32297 DEBRIS DISK
}

\author{
Michael P. Fitzgerald, ${ }^{1,2}$ Paul G. Kalas, ${ }^{1,2}$ and James R. Graham ${ }^{1,2}$ \\ Received 2007 April 16; accepted 2007 July 18
}

\begin{abstract}
We report the detection of a ring of warm dust in the near-edge-on disk surrounding HD 32297 with the Gemini North Michelle mid-infrared imager. Our $N^{\prime}$-band image shows elongated structure consistent with the orientation of the scattered-light disk. The $F_{\nu}(11.2 \mu \mathrm{m})=49.9 \pm 2.1 \mathrm{mJy}$ flux is significantly above the $28.2 \pm 0.6 \mathrm{mJy}$ photosphere. Subtraction of the stellar point-spread function reveals a bilobed structure with peaks $0.5^{\prime \prime}-0.6^{\prime \prime}$ from the star. The disk is detected out to the sensitivity limit at $\sim 1^{\prime \prime}$, and the flux in each lobe is symmetric to within $10 \%$. An analysis of the stellar component of the spectral energy distribution (SED) suggests a spectral type later than A0, in contrast to commonly cited literature values. We fit three-dimensional, single-size grain models of an optically thin dust ring to our image and the SED using a Markov chain Monte Carlo algorithm in a Bayesian framework. The best-fit effective grain sizes are submicron, suggesting the same dust population is responsible for the bulk of the scattered light. The inner boundary of the warm dust is located $0.5^{\prime \prime}-0.7^{\prime \prime}(\sim 65 \mathrm{AU})$ from the star, which is approximately cospatial with the outer boundary of the scattered-light asymmetry inward of $0.5^{\prime \prime}$. The addition of a separate component of larger, cooler grains that provide a portion of the $60 \mu \mathrm{m}$ flux improves both the fidelity of the model fit and consistency with the slopes of the scattered-light brightness profiles. The peak vertical optical depths in our models $\left[\sim(0.3-1) \times 10^{-2}\right]$ imply that grain-grain collisions likely play a significant role in dust dynamics and evolution. Submicron grains can survive radiation pressure blowout if they are icy and porous. Similarly, the inferred warm temperatures $(130-200 \mathrm{~K})$ suggest that ice sublimation may play a role in truncating the inner disk.
\end{abstract}

Subject headings: circumstellar matter — infrared: stars — planetary systems: protoplanetary disks stars: formation — stars: individual (HD 32297)

\section{INTRODUCTION}

The attrition of the primitive remnants of solar system formation replenishes the dust in circumstellar debris disks. The scatteredlight and thermal emission from this dust provide a window into the physical processes governing the evolution of solid material around normal stars, at a time after they have shed their primordial gas and dust envelopes and are transitioning to more mature, nearly dust-free systems (e.g., Backman \& Paresce 1993; Meyer et al. 2007).

HD 32297 ( $d=112_{-12}^{+15}$ pc; Perryman et al. 1997) is a mainsequence star with a recently discovered circumstellar disk. Schneider et al. (2005) first resolved the dust in near-IR scattered light with Hubble Space Telescope (HST) NICMOS (F110W). They detected the near-edge-on inner disk out to $3.3^{\prime \prime}$ ( $\sim 400 \mathrm{AU}$ ) and found a brightness asymmetry inward of $0.5^{\prime \prime}$ and a break in the surface brightness profile at $1.7^{\prime \prime}$. Optical confirmation of the scattered-light disk by Kalas (2005) followed, revealing an extended, asymmetric outer disk extending to $15^{\prime \prime}$ ( $1680 \mathrm{AU}$ ) and suggesting a blue-scattering $R-[\mathrm{F} 110 \mathrm{~W}]$ dust color. Redfield (2007) detected the gaseous component of the disk, finding that HD 32297 exhibits the strongest $\mathrm{Na}$ I absorption of any nearby main-sequence debris disk system.

We report our discovery of spatially resolved emission arising from warm dust surrounding HD 32297 ( 2 2) and note the independent discovery of resolved thermal emission by Moerchen et al. (2007). We examine the morphology of the observed dust emission by removing the direct stellar contribution and determine that the residual structure suggests a model of thermal emission from an optically thin ring. We fit such a model to the data and explore the allowed parameter distribution $(\S 3)$. To conclude,

\footnotetext{
${ }^{1}$ Department of Astronomy, University of California, Berkeley, 601 Campbell Hall, Berkeley, CA 94720.

${ }^{2}$ National Science Foundation Center for Adaptive Optics, University of California, Santa Cruz, CA 95064.
}

we consider the implications of these findings in the context of physical processes shaping the disk's physical structure (§ 4).

\section{OBSERVATIONS}

We observed HD 32297 and HD 20893 with Michelle on the Gemini North telescope on the night of 2006 September 19 (GN-2006B-C-12). HD 20893 serves as the photometric calibrator and point-spread function (PSF) reference. We imaged these stars in the $N^{\prime}$ filter $\left(\lambda_{0}=11.2 \mu \mathrm{m}, \Delta \lambda=2.4 \mu \mathrm{m}\right)$ in a chop-nod sequence with a $15^{\prime \prime}$ throw. Observations were chopped and nodded at a position angle (P.A.) of $120^{\circ}$, chosen to be roughly perpendicular to the scattered-light disk (P.A. $=47.6^{\circ} \pm 1^{\circ}$; G. Schneider 2005 , private communication). Image quality was good ( $N^{\prime}$-band resolution $\sim 0.3^{\prime \prime}$, although terrestrial cirrus contributed to a varying background level which sometimes saturated the detector. The ABBA chop-nod sequences which contained saturated frames were discarded. Of the $188 \mathrm{~s}(23.5 \mathrm{~s})$ of guided, on-source integration time for HD 32297 (HD 20893), $94.0 \mathrm{~s}$ (23.5 s) was used in the final analysis. Each ABBA sequence was processed to remove both the sky and instrumental backgrounds via a double difference. The central, guided images resulting from each ABBA double difference were registered and stacked.

Photometric calibration was performed with HD 20893, which is a Cohen et al. (1999) standard with a zero-air-mass mean $N^{\prime}$-band flux density of $4.29 \pm 0.14 \mathrm{Jy}$ at an isophotal wavelength of $11.23 \mu \mathrm{m}$. The mean air mass during the HD 32297 exposures was $\sim 11 \%$ greater than during the HD 20893 exposures, and we assumed an extinction of $0.172 \mathrm{mag}$ per air mass (corresponding to a mean $N$-band [ $\lambda_{0}=10.0 \mu \mathrm{m}, \Delta \lambda=5 \mu \mathrm{m}$ ] Mauna Kea extinction; Krisciunas et al. 1987) when correcting the measured HD 32297 fluxes.

\section{RESULTS AND ANALYSIS}

The resulting images are shown in Figure 1. The image of HD 32297 exhibits extension consistent with the direction of 


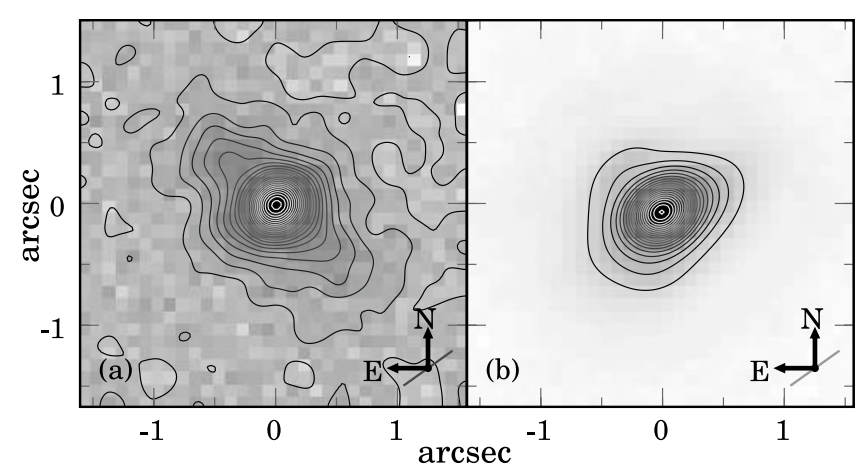

FIG. 1.-(a) Result of our Gemini North Michelle imaging of the HD 32297 debris disk ( $N^{\prime}$ band), showing elongated emission around the star. Contours are spaced according to the $1 \sigma$ background noise level. The elongation P.A. is consistent with the scattered-light disk. The chop direction is indicated by the gray line on the compass. (b) Image of the reference star HD 20893. The contour spacing is selected to match those in $(a)$ relative to the stellar flux.

the scattered-light disk. The extended emission is detected out to the sensitivity limit at $\sim 1^{\prime \prime}$. With a $1.4^{\prime \prime}$ radius aperture and an encircled energy correction derived from the image of HD 20893, we measured the total flux of the star and disk to be $49.9 \pm 2.1 \mathrm{mJy}$. The uncertainty includes the contribution from both the background noise and the zero-point uncertainty derived from HD 20893. As the filter is relatively narrow, a color-correction to this monochromatic flux density was not considered necessary. We place the measurement of total flux in relation to the known spectral energy distribution (SED) in Figure 2.

\subsection{Stellar Properties}

In order to characterize the dust emission, we must (1) estimate the fraction of the observed $N^{\prime}$ flux attributable to the star and (2) estimate the stellar luminosity, which affects the temperature balance of the grains. To these ends, we characterize the star by modeling the optical and near-IR portion of the SED, given by Tycho-2 and Two Micron All Sky Survey (2MASS) photometry (Høg et al. 2000; Skrutskie et al. 2006). For model comparison, we synthesized photometry with the stellar atmospheres of the NextGen grid (Hauschildt et al. 1999). The scaling for the resulting photospheric fluxes, parameterized by $\xi \equiv\left(R_{*} / d\right)^{2}$, was least-squares fit to match the synthetic photometry to the optical/ near-IR data. The choice of model atmosphere parameters is unclear at first glance, since the spectral type of HD 32297 is inconsistent in the literature. It was classified as A0 by A. J. Cannon \& E. C. Pickering in the Henry Draper Catalog, ${ }^{3}$ while it is specified as A5 in the AGK3 Catalog (Heckmann 1975). Assuming no interstellar extinction, $\log g=4.5$, and $[\mathrm{Fe} / \mathrm{H}]=0$, we found that a $T_{\text {eff }}=9600 \mathrm{~K}$ spectrum (appropriate for A0 V) is clearly rejected by the photometry $\left(\chi_{\nu}^{2}=200\right)$, while a $7600 \mathrm{~K}$ NextGen model fit the data best $\left(\chi_{\nu}^{2}=2.5\right)$. The resulting best-fit model is shown in Figure 2.

Can interstellar reddening bias our inferred $T_{\text {eff }}$ ? At $\sim 112 \mathrm{pc}$, the star may be outside the relatively dust-free Local Bubble, which Redfield (2007) notes is expected to extend to $\sim 90 \mathrm{pc}$ in this direction (Lallement et al. 2003). As an empirical test, we fit for the extinction (parameterized by $A_{V}$ ) using the $A_{\lambda} / A_{V}$ law of Fitzpatrick (2004) and assuming $R_{V}=3.1$. The best-fit model, fixing $T_{\text {eff }}=9600 \mathrm{~K}$, gives $A_{V}=0.72 \pm 0.02 \mathrm{mag}$ (formal error)

\footnotetext{
3 Vizier Online Data Catalog, III/135A (A. J. Cannon \& E. C. Pickering, 1993).
}

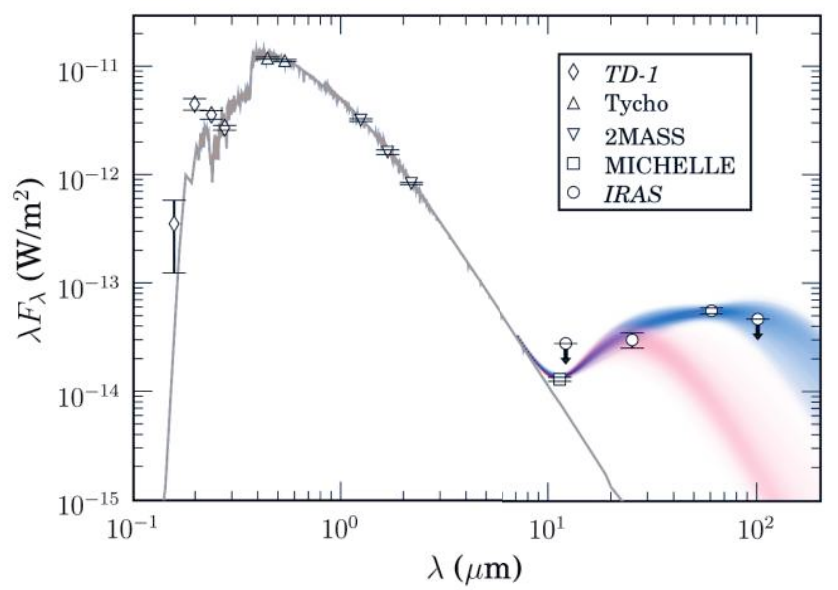

FIG. 2.-SED of the HD 32297 system. The IRAS photometry is from Moór et al. (2006; see also Silverstone 2000). Near-IR data are from 2MASS (Skrutskie et al. 2006), optical data from Tycho-2 (Høg et al. 2000), and UV data from TD-1 (Thompson et al. 1978). A $7600 \mathrm{~K}$ NextGen model photosphere (Hauschildt et al. 1999) with zero foreground extinction was fit to the optical/near-IR photometry. The range of allowed dust emission from model I is shown in blue, while that of model II is shown in red $(\S 3.3)$. Model I fits for the image and all SED points, while model II treats the $60 \mu \mathrm{m}$ measurement as an upper limit, effectively allowing for a separate population of cool dust.

and is rejected with $\chi_{\nu}^{2}=8.5 .^{4}$ When adjusting $T_{\text {eff }}$, the $7600 \mathrm{~K}$ model again minimizes $\chi^{2}$, with a best-fit $A_{V}=0.026 \pm 0.024 \mathrm{mag}$ at this temperature. Thus, we empirically determine that the observations are consistent with zero interstellar extinction.

As an alternative approach to inferring the amount of interstellar dust toward the star, we consider the measurement of the interstellar component of $\mathrm{Na}$ I absorption by Redfield (2007). He measures a column density of $N_{\mathrm{Na}} \sim 10^{12} \mathrm{~cm}^{-2}$. There is considerable scatter in the correlation between $N_{\mathrm{Na} \text { I }}$ and $E(B-V)$, and assuming $R_{V}=3.1$, values of $A_{V}=0.03-0.3 \mathrm{mag}$ are reasonable (Fig. $4 d$ of Hobbs 1974). Since this is consistent with a small amount of extinction, we do not alter our conclusions.

We estimate the flux scaling $\xi=(7.22 \pm 0.14) \times 10^{-20}$ from the best-fit zero-extinction model [after scaling the formal uncertainty by $\left.\left(\chi_{\nu}^{2}\right)^{1 / 2}\right]$. Using this quantity, we calculate the monochromatic flux density $F_{\nu}(11.23 \mu \mathrm{m})=28.2 \pm 0.6 \mathrm{mJy}$. The uncertainties in our estimates of the stellar radius and luminosity are dominated by distance errors, as

$$
L_{*}=4 \pi \xi d^{2} \sigma T_{\mathrm{eff}}^{4} .
$$

Taking the best estimates $\xi=7.22 \times 10^{-20}$ and $d=112 \mathrm{pc}$, we find $R_{*}=1.34 R_{\odot}$ and $L_{*}=5.4 L_{\odot}$. Together, these suggest the literature value of A5 is closer to the "true" classification, although the cool temperature we estimate suggests even later A subclasses. As noted by Schneider et al. (2005) the star lies near the bottom of the A star main sequence in a color-magnitude diagram, a property in common with other young debris disk systems (Jura et al. 1998). Figure 2 of Moór et al. (2006) shows that HD 32297 is relatively underluminous and is positioned toward the red end of the local A star sequence, consistent with our results. A modern high-resolution spectrum can settle the debate over the correct stellar classification.

\footnotetext{
${ }^{4}$ With the addition of $A_{V}$ as a parameter, the number of degrees of freedom $\nu$ has decreased relative to previous fits.
} 


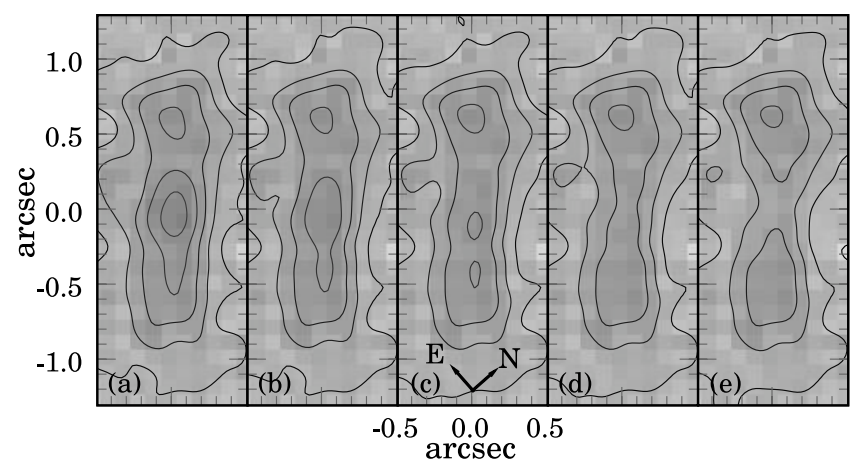

FIG. 3.- Images of the disk with the stellar PSF subtracted for various values of the stellar flux estimate. With $F_{\nu}^{0} \equiv 28.2 \mathrm{mJy}$ and $\sigma \equiv 1.1 \mathrm{mJy}$ (which includes both error in our flux estimate [ $\{3.1]$ and $N^{\prime}$ calibration uncertainty [ $\left.\S 2\right]$ ), (a) $-(e)$ depict subtractions of a central source with $F_{\nu}=\left\{F_{\nu}^{0}-2 \sigma, F_{\nu}^{0}-\sigma, F_{\nu}^{0}\right.$, $\left.F_{\nu}^{0}+\sigma, F_{\nu}^{0}+2 \sigma\right\}$, respectively. As in Fig. 1, contours are spaced according to the $1 \sigma$ background noise level. The bilobed residuals suggest that the system can be modeled as a central star with an optically thin ring of warm material.

Finally, we note the ultraviolet measurements from the $T D-1$ satellite (Thompson et al. 1978) shown in Figure 2. We did not use these data in our photometric fit. The F1965 and F2356 bands ( $\lambda=196.5$ and $235.6 \mathrm{~nm}$, respectively) show excess above the model photosphere. These may be due to line emission- such as $\mathrm{Si}$ III] and $\mathrm{C}$ III] in the former band, and $\mathrm{Fe}$ II in the latter-similar to what is seen in T Tauri stars (e.g., Lamzin 2000a; Valenti et al. 2003). In such stars, the intercombinational lines are likely associated with accretion rather than the chromosphere (Lamzin 2000b). The UV excesses in HD 32297, along with the recent evidence for a gas disk (Redfield 2007), lend indirect support to the contention that this is a young stellar system.

\subsection{PSF Subtraction}

Using the range of $\xi$ estimated in the previous section, we scaled the image of HD 20893 and subtracted it from the that of HD 32297. After PSF subtraction, we find $21.6 \pm 2.2 \mathrm{mJy}$ in non-color-corrected residual flux (Fig. 3). The residuals suggest the observed image (Fig. 1a) is a composite of a bilobed structure and an unresolved central source, whose flux can be fully accounted for by the star. The lobes peak at offsets of $0.5^{\prime \prime}-0.6^{\prime \prime}$ from the star, corresponding to a radius of $\sim 60 \mathrm{AU}$. This suggests an inner edge to the population of grains with optically thin $N^{\prime}$-band emission-both hotter dust closer to the star and the case of optically thick material would fill in the emission at smaller projected separations.

We quantified the degree of asymmetry between the disk lobes with aperture photometry of the PSF-subtracted disk image (Fig. 3c). We used $0.6^{\prime \prime}$ square apertures placed on each lobe, with centers $0.6^{\prime \prime}$ from the stellar centroid. Neglecting an aperture correction and calibration uncertainty, we measured a northeast-southwest flux difference of $0.17 \pm 0.36 \mathrm{mJy}$. As a fraction of the total flux in these apertures, the difference is $(2.0 \pm 4.3) \times 10^{-2}$. We conclude that the disk emission is consistent with symmetry.

\subsection{Modeling}

\subsubsection{Model Construction}

We adopt the model for thermal emission from optically thin dust rings developed by Backman et al. (1992), which assumes particles of effective size $\lambda_{0}$ that radiate as modified blackbodies with emission efficiencies $\epsilon_{\nu}$. We model the three-dimensional emission of the dust $j_{\nu}(\boldsymbol{x})$ by evaluating

$$
\begin{aligned}
& \alpha_{\nu}(\boldsymbol{x})= \begin{cases}\tau_{0}\left(\frac{\varpi}{\varpi_{0}}\right)^{\gamma} f\left[z, h_{0}\left(\frac{\varpi}{\varpi_{0}}\right)^{\eta}\right] & \text { for } \varpi_{0} \leq \varpi \leq \varpi_{1}, \\
0 & \text { otherwise }\end{cases} \\
& \epsilon_{\nu}= \begin{cases}\frac{\lambda_{0}}{\lambda} & \text { if } \lambda>\lambda_{0} \\
1 & \text { otherwise }\end{cases} \\
& T(r)=468\left(\frac{L_{*} / L_{\odot}}{\lambda_{0} / 1 \mu \mathrm{m}}\right)^{1 / 5}\left(\frac{r}{1 \mathrm{AU}}\right)^{-2 / 5} \mathrm{~K}, \\
& j_{\nu}(\boldsymbol{x})=\alpha_{\nu}(\boldsymbol{x}) \epsilon_{\nu} B_{\nu}[T(r)] .
\end{aligned}
$$

Here $\alpha_{\nu}$ is the absorption coefficient, and the function $f(z, h)$ describes the vertical distribution of dust and has scale height $h$. The fiducial values for the vertical optical depth to absorption $\tau_{0}$ and scale height $h_{0}$ are set at the inner edge of the dust annulus, $\varpi_{0}$. We use cylindrical coordinates for positions $\boldsymbol{x}$, with $r^{2}=$ $\varpi^{2}+z^{2}$, and assume azimuthal symmetry.

Some assumptions of grain properties are implicit in equations (2)-(5). The disk is assumed to be optically thin at all radii. The particles are efficient absorbers, but inefficient emitters (eq. [3]). Backman et al. (1992) discuss the relationship between $\lambda_{0}$ and the particle absorption efficiency and size distribution in their Appendix D. They argue that for "dirty ice" dielectric constants and $d n(a) \propto a^{-7 / 2} d a$ collisional-cascade size distribution (Dohnanyi 1969), the minimum grain size is an uncertain $a_{\min } \sim \lambda_{0} / 6$. With these assumptions, the model contains only minimal information regarding grain characteristics. While we ignore the effects of grain composition with this approach, the absence of evidence for grain mineralogy (e.g., mid-IR spectroscopy) renders consideration of various compositions premature. Future work using more advanced models with explicit dependence on grain sizes and mineralogy may produce results that are significantly different from ours.

For simplicity, we assume the vertical density distribution $f$ is Gaussian, with width $\sigma=h_{0}=10(d / 112 \mathrm{pc})$ AU fixed at all radii $(\eta=0)$. Because we do not measure a significant brightness asymmetry $(\S 3.2)$, we assume the disk is spatially centered on the star. The distance $d$ is a "nuisance" parameter not directly related to the disk architecture, and in the interest of independence we define $\theta_{0} \equiv \varpi_{0} / d$ (likewise for $\theta_{1}$ ). The remaining free parameters are $\theta_{0}, \theta_{1}, \lambda_{0}$, vertical optical depth to absorption $\tau_{0} \equiv \tau_{\perp}^{\mathrm{abs}}\left(\varpi_{0}\right)$, (subpixel) stellar centroid $\boldsymbol{x}_{*}$, stellar flux (parameterized by $\xi$; $\S 3.1$ ), disk inclination $i$, and P.A. The stellar luminosity is given by equation (1). There are 11 degrees of freedom within the set of model parameters $\Theta=\left\{d, \boldsymbol{x}_{*}\right.$, P.A., $\left.i, \xi, \gamma, \theta_{0}, \theta_{1}, \tau_{0}, \lambda_{0}, h_{0}, \eta\right\}$.

We construct the density distribution on a three-dimensional grid, with spatial resolution of $0.05^{\prime \prime}=5.6(d / 112 \mathrm{pc}) \mathrm{AU}$, which is half the spatial sampling of the Michelle detector $\left(0.1^{\prime \prime} \operatorname{pixel}^{-1}\right)$. The emission coefficient $j_{\nu}$ (eq. [3]) is sampled at the grid points and numerically integrated along each line of sight. As in the simple subtraction in $\S 3.2$, the PSF is derived from the image of HD 20893 (Fig. 1b). We upsample the PSF to match the grid resolution, shift it according to $\boldsymbol{x}_{*}$, and convolve it with the disk model's integrated emission. These procedures take place in the Fourier domain with their Fourier-equivalent operations via the convolution and shift theorems. We transform back to the image domain and bin the result to the resolution of the instrument (a factor of 2 in each dimension). 


\subsubsection{Fitting Process}

Given the model disk, how do we characterize the range of model parameters allowed by the data? As we have only limited information (e.g., the thermal emission resolved in a single band), we expect that degeneracies will exist between some parameters (such as $\lambda_{0}$ and $\tau_{0}$ ). Furthermore, because equations (1)-(5) contain significant nonlinearities, we expect non-Gaussianity in the joint distribution of allowed parameters. Finally, this problem contains nuisance parameters (e.g., $d$ ) which are unimportant when considering the physical architecture of the disk. These characteristics, combined with a desire to incorporate prior knowledge, suggest a Bayesian approach to model fitting. The range of disk architectures allowed by our simple model can be described by the joint posterior distribution of model parameters, namely,

$$
p(\Theta \mid D, I) \propto p(D, \Theta \mid I)=p(D \mid \Theta, I) p(\Theta \mid I) .
$$

The posterior distribution of parameters is proportional to the likelihood, $p(D \mid \Theta, I)$, times the prior, $p(\Theta \mid I)$. Here $D$ represents the observational data and $I$ represents our background information. In this framework, the best-fit model is one whose parameters maximize the posterior distribution (so-called maximum a posteriori fitting). The Bayesian approach has previously been used in modeling mid-IR images of debris disks (e.g., Koerner et al. 1998; Wahhaj et al. 2003). A more general comparison of Bayesian and frequentist methods in the context of common astronomical problems is given in Loredo (1992).

Brute-force characterization of this distribution is not computationally efficient. The large number of model parameters ensures that the posterior has a relatively high dimensionality, and gridbased schemes for mapping the posterior require many evaluations of the model. Instead, we turn to Markov chain Monte Carlo (MCMC) methods to more efficiently explore the parameters' joint posterior distribution. We note that these advanced statistical techniques stand in contrast to the relative simplicity of our model; our goal of measuring the ranges of allowed disk architectures motivates the expenditure of computational resources in the exploration of parameter space rather than the complexity of the underlying disk model.

The MCMC framework uses a Markov chain, consisting of a series of states $\left\{\Theta_{n}\right\}$, to explore the parameter space and sample the posterior. A requirement of MCMC methods is an algorithm for randomly choosing states in the Markov chain. Given a state $\Theta_{n}$, the Metropolis-Hastings algorithm provides a general-purpose method for randomly choosing the subsequent state $\Theta_{n+1}$ based on a candidate transition function (commonly Gaussian). Often this algorithm is used in conjunction with the Gibbs sampler, which provides a prescription for choosing which of the model variables to change in each transition. With the Metropolis-Hasting algorithm choosing states, the chain will eventually reach convergence. A key property of converged chains is that the distribution of states approximates the posterior distribution. The use of MCMC in astrophysical data analysis has grown in recent years; a summary of this technique and an application to quantify uncertainty in orbit fitting is given by Ford (2005).

When addressing questions about the physical properties of the disk, we are sometimes interested only in the range of a subset of parameters allowed by the model. In the Bayesian framework, the distribution of a parameter (or joint distribution of a subset of parameters) is obtained through marginalization. The posterior is integrated over the variables not of interest. For example, to obtain the joint marginal distribution of $\tau_{0}$ and $\lambda_{0}$, we integrate over the other variables $\Theta^{\prime}=\left\{d, \boldsymbol{x}_{*}\right.$, P.A., $\left.i, \xi, \gamma, \theta_{0}, \theta_{1}, h_{0}, \eta\right\}$,

$$
p\left(\tau_{0}, \lambda_{0} \mid D, I\right)=\int p(\Theta \mid D, I) d \Theta^{\prime} .
$$

Since we have obtained an MCMC chain, this integral is trivialthe joint marginal distribution is simply the joint distribution of the variables' samples, in this case the distribution of $\left\{\tau_{0, n}, \lambda_{0, n}\right\}$.

Having chosen the sampling scheme, we must define the likelihood and prior distributions for our model parameters. We calculate the likelihood by subtracting the model image from the observed emission presented in Figure $1 a$. We compute $\chi_{\mathrm{im}}^{2}$ for the image fit using the background noise level, and we add the $\chi_{\text {SED }}^{2}$ arising from the IRAS SED measurements (including upper limits). The likelihood is then $p(D \mid \Theta, I) \propto \exp \left[-\left(\chi_{\mathrm{im}}^{2}+\chi_{\mathrm{SED}}^{2}\right) / 2\right]$.

We have several priors whose product constitutes $p(\Theta \mid I)$. We use normal priors for the Hipparcos parallax (8.92 \pm 1.05 mas; a proxy for $d$ ) and the stellar flux factor $\xi(\S 3.1)$. We also apply a log-uniform prior to $\lambda_{0}$, with limits of $1 \mathrm{~nm}$ to $1 \mathrm{~mm}$, as well as a uniform prior on $\gamma$, ranging from -4 to 4 . The prior for the outer disk extent $\theta_{1}$ is taken to be uniform from $\theta_{0}$ to $200^{\prime \prime}$. With these likelihood and prior functions, we ran a total of six chains using the Metropolis-Hastings algorithm with the Gibbs sampler. Each chain contained $10^{4}$ samples. The first $10^{3}$ samples were discarded, as the candidate transition function proposal variance for each parameter was adjusted during this "burn-in" period. We expect the Markov chains have converged because no gross deviations exist when comparing the marginal distributions of each parameter across all chains.

In general, the convergence rate and outcome of fitting processes are dependent on starting conditions. Rather than input handpicked parameters to the MCMC algorithm, we first apply a genetic algorithm (for global optimization) followed by a Levenberg-Marquardt least-squares fit (for local optimization) to find a model of the image with suitable parameters. During this process, the (nuisance) distance is fixed at 112 pc. Examination of the marginal distributions of each parameter shows that the posterior distribution in the region of the global maximum is smooth. As a check for other maxima, we drew 500 randomly distributed samples of $\Theta$ over a wide distribution and performed a leastsquares fit with each sample as the starting condition. All fits converged on the same maximum, which suggests the absence of lesser maxima in the posterior distribution.

$$
\text { 3.3.3. Results }
$$

Using the ring model presented in $\S 3.3 .1$, we fit the $N^{\prime}$-band image and in the SED the 25 and $60 \mu \mathrm{m}$ points while respecting the 12 and $100 \mu \mathrm{m}$ upper limits (model I). Confidence intervals for the marginal posterior parameter distributions are given in Table 1. We are able to obtain a reasonable fit to the image, although the model tends to overpredict the 25 and $100 \mu$ m fluxes (Fig. 2). With the parameters that maximize the posterior, the $\chi^{2}$ for the image is 912 (with $32^{2}$ pixels), while the $\chi^{2}$ for the IRAS SED is 3.3 (with two data points and two upper limits).

The marginal distributions for the position angle and inclination are approximately normal, with P.A. $=44.4^{\circ} \pm 1.3^{\circ}$ and $i=90.0^{\circ} \pm 2.4^{\circ}$. The P.A. is marginally inconsistent with the direction of the inner scattered-light disk, although this should not be taken as evidence of separate disks, since we did not calibrate the detector orientation. We note that the range of allowed inclinations may be affected by our choice of disk scale height.

The SED data at $\geq 25 \mu \mathrm{m}$ constrain the values of the outer radius $\theta_{1}$ and density power-law index $\gamma$. From the marginal 
TABLE 1

Best-Fit Model Parameters

\begin{tabular}{|c|c|c|c|}
\hline Parameter & Model I & Model II & Notes \\
\hline \multicolumn{4}{|c|}{ Free Parameters } \\
\hline P.A. (deg) ................... & $44.5_{-2.5}^{+2.6}$ & $44.5 \pm 2.5$ & \\
\hline$i(\mathrm{deg}) \ldots \ldots$ & $90.0 \pm 4.7$ & $90.0 \pm 4.8$ & \\
\hline$\xi$ & $1.02 \pm 0.03$ & $1.03 \pm 0.03$ & $\times 7.22 \times 10^{-20}$ \\
\hline$\gamma$ & $0.07 \pm 0.22$ & $-2.1_{-1 .}^{+2.2}$ & $\mathrm{a}$ \\
\hline$\theta_{0}(\operatorname{arcsec}) \ldots \ldots \ldots \ldots \ldots$ & $0.54_{-0.07}^{+0.05}$ & $0.65_{-0.09}^{+0.06}$ & \\
\hline$\theta_{1}(\operatorname{arcsec})$ & $>33$ & $>1.8$ & \\
\hline $\log \tau_{0} \ldots \ldots \ldots \ldots \ldots \ldots \ldots$ & $-2.57_{-0.30}^{+0.24}$ & $-1.94_{-0.49}^{+0.27}$ & \\
\hline $\log \left(\lambda_{0} / 1 \mu \mathrm{m}\right) \ldots \ldots \ldots \ldots$ & $-0.65_{-0.27}^{+0.21}$ & $-0.41_{-0.22}^{+0.15}$ & \\
\hline
\end{tabular}

Dependent or Fixed Parameters

\begin{tabular}{|c|c|c|}
\hline$h_{0}(\mathrm{AU})$ & $10(d / 112 \mathrm{pc})$ & $10(d / 112 \mathrm{pc})$ \\
\hline$\eta$ & 0 & 0 \\
\hline$\varpi_{0}(\mathrm{AU}) \ldots \ldots \ldots \ldots \ldots \ldots$ & $61_{-13}^{+20}$ & $73_{-16}^{+23}$ \\
\hline$\varpi_{1}(\mathrm{AU}) \ldots \ldots \ldots \ldots \ldots \ldots \ldots$ & $>3700$ & $>210$ \\
\hline$T\left(\varpi_{0}\right)(\mathrm{K}) \ldots \ldots \ldots \ldots$ & $171_{-19}^{+31}$ & $142_{-11}^{+21}$ \\
\hline
\end{tabular}

Notes.-Confidence intervals are $95 \%$ for marginal posterior distributions. P.A. is the position angle, $i$ the inclination, $\xi=\left(R_{*} / d\right)^{2}$ the stellar flux parameter, $\gamma$ the surface density power-law index, $\theta_{0,1}$ the disk inner and outer edges, respectively, in angular units, $\tau_{0}$ the vertical optical depth to absorption at the inner edge, $\lambda_{0}$ the effective grain size, $h_{0}$ the scale height at the inner edge, $\eta$ the scale height power law (see eq. [2]), $\varpi_{0,1}$ the inner and outer edges, respectively, in spatial units, and $T\left(\varpi_{0}\right)$ the dust temperature at the inner edge.

a The lower limit in model II is set by the prior.

parameter distribution, we find $\theta_{1} \gtrsim 30^{\prime \prime}$, which is farther than the scattered-light disk has been detected (15"; Kalas 2005). The model also requires $\gamma \simeq 0$, which places a large amount of cool material in the outer disk. With $\lambda_{0} \simeq 0.2 \mu \mathrm{m}$, giving $a_{\min } \ll 1 \mu \mathrm{m}$, we can infer this to be the same population giving a blue $R$ [F110W] color, expected for small grains approaching the Rayleigh regime (Kalas 2005). We note that Rayleigh grains scatter light quasi-isotropically, in the sense that the first moment of the scattering phase function $(g \equiv\langle\cos \theta\rangle)$ is zero. For an edge-on, wedge-shaped disk $(\eta=1)$ of a power-law distribution of isotropic scatterers, the midplane surface brightness profile at projected distance $b$ is $\propto b^{\gamma-1}$. Under these assumptions, model I predicts a brightness profile power-law index of $\sim-1$, which is shallower than the measured indices $(-2.7$ to -3.7 ; Schneider et al. 2005; Kalas 2005). This suggests that the long-wavelength SED is not produced by a relatively flat distribution out to large radii, but rather by a separate population of grains. Similar populations have been invoked for other systems (e.g., in $\beta$ Pic and AU Mic; Backman et al. 1992; Fitzgerald et al. 2007).

The large spatial extent of model I and its inconsistency with the scattered-light profile power law suggest that we should amend the model with an additional grain population. One possibility would be to maintain a distribution of small grains, similar to model I, to produce the $N^{\prime}$-band image and $25 \mu \mathrm{m}$ flux, and add larger, cooler grains that reproduce the $60 \mu \mathrm{m}$ flux but contribute little to the shorter wavelength emission. This would require additional parameters; modeling a single ring of large grains requires the ring distance and the grains' size and number. In the interest of computational simplicity, we choose not to explicitly parameterize the larger grains in model II. Instead, we implicitly allow for this population by relaxing the SED fit, requiring only that the $60 \mu \mathrm{m}$ flux from the grain population producing the spatially resolved emission does not exceed the IRAS measurement. The unmodeled population is presumed to supplement the small grain emission to match the observed $60 \mu \mathrm{m}$ flux. The larger grains are

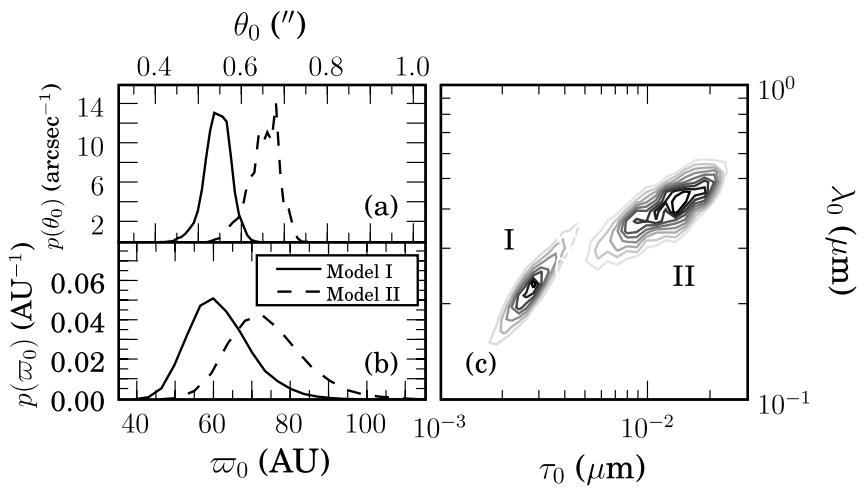

FIG. 4.-Marginal posterior distributions of a subset of model parameters. (a)(b) Probability density of the location of the inner disk edge in angular $\left(\theta_{0}\right)$ and physical $\left(\varpi_{0}\right)$ units, respectively. The ordinates of these plots are matched assuming a distance of $112 \mathrm{pc}$, and the differences evident between the distributions highlight the value of the joint estimation of all parameters. (c) Joint marginal posterior distribution for effective grain size $\lambda_{0}$ and vertical optical depth to absorption at the inner edge $\tau_{0}$.

assumed to emit little at $25 \mu \mathrm{m}$. In effect, we treat the $60 \mu \mathrm{m}$ detection as an upper limit.

After running the MCMC chains with the same procedure as before, we find that model II reproduces the $N^{\prime}$-band image and the $25 \mu \mathrm{m}$ flux. With the parameters that maximize the posterior, the $\chi^{2}$ for the image is 902 , while the $\chi^{2}$ for the IRAS SED is 0.4 (with one data point and three upper limits). One drawback to our decision to avoid explicitly parameterizing the large grains in this model is that it precludes rigorous comparison of the goodness of fit to that of model I. ${ }^{5}$ However, we expect that when combined with scattered-light data, this model will be favored because of its ability to include surface density gradients with $\gamma<0$.

Model II produces less stringent requirements on $\gamma$ than from model I, favoring steeper drop-off, although flat distributions are not excluded. The distributions of the inner radii, in terms of both angular $\left(\theta_{0}\right)$ and physical $\left(\varpi_{0}\right)$ variables, are given in Figures $4 a$ and $4 b$.

To give a basic example of possible parameters for the unmodeled large-grain component, we added the flux from a single ring of large grains at $80 \mathrm{AU}$ to the best-fit model II. We found that $10^{29} \mathrm{~cm}^{2}$ of $\lambda_{0}=85 \mu \mathrm{m}$ grains were able to reproduce to $60 \mu \mathrm{m}$ flux without strongly affecting the $25 \mu \mathrm{m}$ component from the small grains. These large grains have a temperature of $50 \mathrm{~K}$ according to equation (4). These large-grain parameters are only a single sample in a range of possibilities, and we note that they are expected to be strongly covariant without additional observations of the far-IR and submillimeter flux.

In both models, the effective grain size $\lambda_{0}$ and the fiducial optical depth $\tau_{0}$ are covariant. This is evident in their marginal joint distributions, shown in Figure $4 c$. The relaxation of the far-IR SED requirement allows for a broader range for these parameters in model II. We stress that joint estimation of parameters is frequently necessary in disk models. For example, in scattered-light modeling of AU Mic, Graham et al. (2007) revealed a strong degeneracy between scattering asymmetry and the surface density power law, which was broken by the independent constraint from polarization measurements.

\footnotetext{
5 This is usually done by comparing the "evidence" for each model, through the ratio $\int p\left(D, \Theta_{\mathrm{I}} \mid\right.$ model $\left.\mathrm{I}, I\right) d \Theta_{\mathrm{I}} / \int p\left(D, \Theta_{\mathrm{II}} \mid\right.$ model II,$\left.I\right) d \Theta_{\mathrm{II}}$, with a possible additional factor representing prior preference for one model over the other (e.g., Sivia 2006).
} 
In principle, the choice of the prior $p(\Theta \mid I)$ can affect the distributions of parameters given in Table 1. A parameter's distribution is sensitive to the prior if it is not dominated by the likelihood term in equation (6). We explored the sensitivity to choice of prior by comparing the marginal distributions of each parameter with its prior. In general, both models I and II show similar sensitivity to priors. In each model, the marginal posterior distribution of $\xi$ is roughly Gaussian in shape, but its mean is offset from the mean of the Gaussian prior. In this case, both the likelihood and prior play a significant role in shaping the posterior distribution. The marginal posterior distribution of the distance $d$ is completely dominated by the prior derived from the Hipparcos parallax. This is unsurprising, since we did not use a prior for the stellar radius or luminosity that might serve to constrain the distance. The marginal posterior for the outer radius $\theta_{1}$ largely follows the flat prior (out to $200^{\prime \prime}$ ), although it has an inner cutoff set by the likelihood function. Thus, the lower limits listed in Table 1 are relatively insensitive to the prior. The marginal posterior of $\gamma$ is well constrained by the likelihood function in model I, but in model II the prior affects the posterior. In the latter model, the distribution shows an upper limit cutoff near $\gamma=0$ and a peak near $\gamma=-2$. However, the distribution is smooth down to the lower cutoff set by the prior at $\gamma=-4$. Therefore, the uniform prior for $\gamma$ plays a role in shaping the posterior distribution in model II, and the likelihood function is only able to exclude values in the upper end of the range. For all other variables, the likelihood function is sharply peaked compared to the relatively flat priors; therefore, their results are insensitive to our choice of prior.

\section{DISCUSSION}

The spatial locations and inferred sizes of the thermally emitting grains are largely consistent with the dust seen in scattered light. Schneider et al. (2005) find that the scattered-light disk is symmetric for $0.5^{\prime \prime}<\theta<1.7^{\prime \prime}$, which overlaps with the symmetric mid-IR emission we measure in $\S 3.2$. The inner edge of the warm dust ring $\left(\theta_{0} \simeq 0.5^{\prime \prime}-0.7^{\prime \prime}\right)$ corresponds to the outer boundary of the scattered-light brightness asymmetry seen inward of $0.5^{\prime \prime}$. A comparison of the northeast and southwest F110W surface brightness profiles shows the southwest ansa is $\sim 2$ times brighter than the northeast at $0.5^{\prime \prime}$. By integrating the disk light in each ansa from $\theta>0.3^{\prime \prime}$, Schneider et al. find the total northeast (southwest) emission to be $1.67 \pm 0.57 \mathrm{mJy}(3.14 \pm 0.57 \mathrm{mJy})$, which corresponds to a northeast-southwest fractional flux difference of $-0.31 \pm 0.18$. In comparison to the scattered light, in the $N^{\prime}$-band image we do not find an increasing brightness trend for the southwest ansa inward of $0.6^{\prime \prime}$, nor do we find evidence for asymmetry in this region. How can the emission appear asymmetric in scattered light, but symmetric in thermal emission? One possibility is that the grains responsible for the scattered-light asymmetry reside inward of the warm ring. In this case, they must have properties that differ from the majority of grains in the inner disk, which scatter light in the symmetric component. If the asymmetrically distributed grains have a significantly higher albedo or larger average size, they may not be apparent in the thermal emission. Future observations of scattered light at different wavelengths may constrain such changes in grain properties with position. Another possibility is that the asymmetry is produced by a density enhancement at sufficient distance from the star such that its grains do not produce significant $N^{\prime}$-band emission relative to the warm ring.

For $\lambda_{0} \simeq 0.1-0.5 \mu \mathrm{m}$ and reasonable assumptions on grain composition and size distribution $(\S 3.3 .1)$, we find $a_{\min } \sim$ $\lambda_{0} / 6 \simeq 0.02-0.1 \mu \mathrm{m}$. As noted in $\S 3.3 .3$, in the $R$ and F110W bands, submicron grains will scatter blue as their sizes approach the Rayleigh regime, consistent with the blue color of dustscattered light inferred by Kalas (2005). This lends support to the contention that the scattered-light images and thermal image presented here are probing similar populations of grains. Simultaneous modeling of the scattered-light and thermal emission, deferred for future work, has the potential to strongly constrain grain sizes, locations, and composition for the bulk of the inner disk.

These observations are a stepping stone to understanding the nature of the disk and source of its structure. However, we lack direct evidence for the age of the system, which complicates such analysis. Based on the stellar distance and Galactic space motion, Kalas (2005) argues for an age of 30 Myr based on the system's possible association with the Gould Belt or recent star formation in the Taurus-Auriga association. As we show in the following calculations, this age indicates that the grains in the inner disk are likely not primordial and must be replenished. In the next subsection, we seek to estimate orders of magnitude for processes governing grain production and removal for the sizes and spatial locations in the innermost disk.

\subsection{Grain Dynamics}

Assuming the radiative coupling efficiency averaged over the stellar spectrum $Q_{\text {rad }}=2$, a grain density of $2 \mathrm{~g} \mathrm{~cm}^{-3}$, and $M_{*}=$ $1.8 M_{\odot}$, the fiducial radiation pressure blowout size is $a_{\text {blow }} \sim$ $3 \mu \mathrm{m}$ (Burns et al. 1979). In the absence of forces other than radiation and gravity, grains smaller than this size are removed from the system on the free-fall timescale, $\sim 10^{4} \mathrm{yr}$. At first glance, this would imply that production of submicron grains must be extremely rapid. However, our conversion from $\lambda_{0}$ to the geometric size $a_{\min }$ is quite uncertain and may be too small by a factor of $\sim 30$ (if the grains are weakly absorbing and have a size distribution steeper than $d n \propto a^{-3.5} d a$; Backman et al. 1992). Another possibility, which maintains consistency with submicron scattered-light grains, is that drag forces can increase the residence time of the grains undergoing ejection. Finally, we note that $a_{\mathrm{blow}}$ is not a strict lower limit to the steady state size distribution. As noted by Burns et al. (1979), very small grains (sizes much less than the peak wavelength of stellar radiation) couple inefficiently to the radiation field, such that $Q_{\text {rad }} \ll 1$ and the radiation force cannot overcome gravity. For such small grains, composition and porosity can play a crucial role in the residence time due to their effects on the optical constants. For example, Grigorieva et al. (2007) show in their Figure 1 that around an A5 V star, silicate grains are blown out regardless of size or porosity. In contrast, the smallest icy grains can remain bound. Compact icy grains have a blowout size of $3 \mu \mathrm{m}$, whereas $a_{\text {blow }}$ for grains with $80 \%$ porosity drops to $0.7 \mu \mathrm{m}$. Because of their small $Q_{\mathrm{rad}}$, the radiation pressure on $80 \%$ porous, icy grains smaller than $0.05 \mu \mathrm{m}$ is insufficient to overcome gravity. This cutoff size decreases to $\sim 0.02 \mu \mathrm{m}$ for more compact icy grains. We conclude that our model grain sizes may be compatible with the steady state size distribution arising from radiation pressure and note that this mechanism provides a natural separation between a population of small ( $a \lesssim 0.05 \mu \mathrm{m})$ grains and a population of grains larger than $a_{\text {blow }}$.

Collisions may play a significant role in the lifetimes of grains in the inner disk, as fragments from a catastrophically dispersed grain can be rapidly removed via radiation pressure. For a loweccentricity disk of single-size particles, the timescale between mutual collisions is $t_{\text {coll }} \sim\left(\Omega \tau_{\perp}^{\text {geo }}\right)^{-1}$, where $\Omega$ is the orbital frequency and $\tau_{\perp}^{\text {geo }}$ is the geometric vertical optical depth. We 
find $t_{\text {coll }} \sim 6 \times 10^{4}(r / 60 \mathrm{AU})^{3 / 2}\left(\tau_{\perp}^{\text {geo }} / 10^{-3}\right)^{-1}$ yr. Calculation of this timescale from our models is complicated by the unknown absorption efficiency, as $\tau_{\perp}^{\text {geo }}\left(\varpi_{0}\right)=\tau_{0} / Q_{\text {abs }}$. We also note that the timescale for destructive collisions is likely different from the above $t_{\text {coll }}$, and it must account for the unknown grain size and velocity distributions. The presence of a gas disk can damp relative velocities, increasing the timescale for destructive collisions. These damping forces can also cause grains to settle in the midplane, causing the disk to be very thin in vertical extent (e.g., Garaud et al. 2004). Observations of the disk scale height may constrain the presence of gas and its effect on collisional timescales. Our models in $\S 3.3$ show that the mid-IR image is consistent with an edge-on, vertically unresolved disk. More work is needed in modeling scattered-light images (at higher spatial resolution) to determine if the apparent disk thickness is the result of vertical extent or inclination effects. Alternately, it may be possible to constrain the gas density through modeling of the dust density's radial structure, as has been done for $\beta$ Pic (Thébault \& Augereau 2005). Regardless, future dynamical models of the inner disk must make a detailed accounting of grain-grain collisions.

Drag forces can decrease the periastra of grain orbits, filling the inner disk with material. Assuming circular orbits, the PoyntingRobertson drag timescale at radius $r$ is $t_{\mathrm{PR}} \sim 10^{6}(a / 1 \mu \mathrm{m}) \times$ $\left(\rho / 2 \mathrm{~g} \mathrm{~cm}^{-3}\right)(r / 60 \mathrm{AU})^{2}\left(Q_{\mathrm{rad}} / 2\right)^{-1}$ yr. This is significantly longer than $t_{\text {coll }} \lesssim 10^{5} \mathrm{yr}$, suggesting Poynting-Robertson drag is dynamically unimportant for the radii of warm dust emission (although it may allow some mid-IR-emitting dust in systems without planets; see Wyatt 2005). The detection of a potentially massive gas disk by Redfield (2007) suggests that gas drag may affect the grains in this system. As noted above, the role of gas drag in the inner disk is difficult to estimate due to our ignorance of the gas disk's density distribution and physical state, and no physical features (such as sharp outer edges of dust rings or midplane settling) currently provide such indications (e.g., Takeuchi \& Artymowicz 2001; Besla \& Wu 2007).

An interesting physical result from our models is the warm temperatures of the effective grains at the inner rim of the dust annulus $\left[T\left(\varpi_{0}\right) \simeq 130-200 \mathrm{~K}\right]$. At these temperatures, the sublimation of water ice in small grains is efficient $\left(t_{\text {sub }} \lesssim 1 \mathrm{yr}\right)$, suggesting no water is present in grains at this distance from the star. However, this process is a strong function of temperature (see eq. [16] of Backman \& Paresce 1993) and therefore stellar distance (eq. [4]). At a distance of $r=2 \varpi_{0}$, a $0.2 \mu \mathrm{m}$ water ice grain has $t_{\text {sub }} \sim 1$ Myr. This raises the possibility that, rather than being the location of grain creation and outward diffusion, the inner edge of the warm dust disk is the destruction site for icy inspiraling grains. For this to be the case, drag forces must overcome radiation pressure and destructive collisions for a significant population of icy grains.

The inference of the physical processes responsible for the disk structure (and that govern its evolution) is unclear. Direct spectroscopic evidence of the stellar age is still needed. Furthermore, the spatial distribution of the gas disk is an important direction for future observations. This system is attractive for the direct detection of gas emission, similar to observations of $\beta$ Pic (Thi et al. 2001; Olofsson et al. 2001; Brandeker et al. 2004). The next modeling steps should combine the available data, including the resolved scattered light, thermal emission, and SED. More detailed calculations can reveal the processes responsible for the disk structure, in a manner similar to that developed for AU Mic by Strubbe \& Chiang (2006).

\subsection{Conclusions}

We have (1) spatially resolved the thermal emission from the warm inner disk around HD 32297 in the $N^{\prime}$ band, (2) found that the stellar SED is inconsistent with the temperature and luminosity of A0, instead favoring a cooler, less luminous star, (3) found that the observed $N^{\prime}$ emission with the stellar PSF subtracted suggests a symmetric, optically thin ring model, (4) modeled the thermal emission (including IRAS SED data) with an annulus consisting of a single population of efficiently absorbing, inefficiently radiating grains, (5) determined that a separate population producing the $60 \mu \mathrm{m}$ emission improves the fit to the SED and the consistency with the scattered-light disk, and (6) identified the possibility that ice sublimation may play a significant role in the destruction of grains in the warm inner disk.

We would like to thank Scott Fisher and Kevin Volk for their helpful observing support. This publication makes use of data products from the Two Micron All Sky Survey, which is a joint project of the University of Massachusetts and the Infrared Processing and Analysis Center/California Institute of Technology, funded by NASA and the NSF. This work is based on observations obtained at the Gemini Observatory, which is operated by AURA, Inc., under a cooperative agreement with the NSF on behalf of the Gemini partnership. The authors acknowledge the significant cultural role that the summit of Mauna Kea has always had within the indigenous Hawaiian community. This work was supported in part by the NSF Science and Technology Center for Adaptive Optics, managed by the University of California at Santa Cruz under cooperative agreement AST 98-76783.

\section{REFERENCES}

Backman, D. E., \& Paresce, F. 1993, in Protostars and Planets III, ed. E. H.

Levy \& J. I. Lunine (Tucson: Univ. Arizona Press), 1253

Backman, D. E., Witteborn, F. C., \& Gillett, F. C. 1992, ApJ, 385, 670

Besla, G., \& Wu, Y. 2007, ApJ, 655, 528

Brandeker, A., Liseau, R., Olofsson, G., \& Fridlund, M. 2004, A\&A, 413, 681

Burns, J. A., Lamy, P. L., \& Soter, S. 1979, Icarus, 40, 1

Cohen, M., Walker, R. G., Carter, B., Hammersley, P., Kidger, M., \& Noguchi, K. 1999, AJ, 117, 1864

Dohnanyi, J. S. 1969, J. Geophys. Res., 74, 2431

Fitzgerald, M. P., Kalas, P. G., Duchêne, G., Pinte, C., \& Graham, J. R. 2007, ApJ, in press

Fitzpatrick, E. L. 2004, in ASP Conf. Ser. 309, Astrophysics of Dust, ed. A. N. Witt, G. C. Clayton, \& B. T. Draine (San Francisco: ASP), 33

Ford, E. B. 2005, AJ, 129, 1706

Garaud, P., Barrière-Fouchet, L., \& Lin, D. N. C. 2004, ApJ, 603, 292

Graham, J. R., Kalas, P. G., \& Matthews, B. C. 2007, ApJ, 654, 595

Grigorieva, A., Artymowicz, P., \& Thébault, P. 2007, A\&A, 461, 537

Hauschildt, P. H., Allard, F., \& Baron, E. 1999, ApJ, 512, 377

Heckmann, O. 1975, AGK 3: Star Catalogue of Positions and Proper Motions North of $-2.5^{\circ}$ Declination, ed. W. Dieckvoss (Hamburg-Bergedorf: Hamburger Sternw.)

Hobbs, L. M. 1974, ApJ, 191, 381

Høg, E., et al. 2000, A\&A, 355, L27

Jura, M., Malkan, M., White, R., Telesco, C., Pina, R., \& Fisher, R. S. 1998, ApJ, 505, 897

Kalas, P. 2005, ApJ, 635, L169

Koerner, D. W., Ressler, M. E., Werner, M. W., \& Backman, D. E. 1998, ApJ, $503, \mathrm{~L} 83$

Krisciunas, K., et al. 1987, PASP, 99, 887

Lallement, R., Welsh, B. Y., Vergely, J. L., Crifo, F., \& Sfeir, D. 2003, A\&A, 411, 447

Lamzin, S. A. 2000a, Astron. Lett., 26, 589

. 2000b, Astron. Rep., 44, 323

Loredo, T. 1992, in Statistical Challenges in Modern Astronomy, ed. E. Feigelson \& G. Babu (Berlin: Springer), 275 
Meyer, M. R., Backman, D. E., Weinberger, A. J., \& Wyatt, M. C. 2007, in Protostars and Planets V, ed. B. Reipurth, D. Jewitt, \& K. Keil (Tucson: Univ. Arizona Press), 573

Moerchen, M. M., Telesco, C. M., De Buizer, J. M., Packham, C., \& Radomski, J. T. 2007, ApJ, 666, L109

Moór, A., Ábrahám, P., Derekas, A., Kiss, C., Kiss, L. L., Apai, D., Grady, C., \& Henning, T. 2006, ApJ, 644, 525

Olofsson, G., Liseau, R., \& Brandeker, A. 2001, ApJ, 563, L77

Perryman, M. A. C., et al. 1997, A\&A, 323, L49

Redfield, S. 2007, ApJ, 656, L97

Schneider, G., Silverstone, M. D., \& Hines, D. C. 2005, ApJ, 629, L117

Silverstone, M. D. 2000, Ph.D. thesis, Univ. California, Los Angeles

Sivia, D. S. 2006, Data Analysis: A Bayesian Tutorial (2nd ed.; New York: Oxford Univ. Press), chap. 4
Skrutskie, M. F., et al. 2006, AJ, 131, 1163

Strubbe, L. E., \& Chiang, E. I. 2006, ApJ, 648, 652

Takeuchi, T., \& Artymowicz, P. 2001, ApJ, 557, 990

Thébault, P., \& Augereau, J.-C. 2005, A\&A, 437, 141

Thi, W. F., et al. 2001, Nature, 409, 60

Thompson, G. I., Nandy, K., Jamar, C., Monfils, A., Houziaux, L., Carnochan, D. J., \& Wilson, R. 1978, Catalogue of Stellar Ultraviolet Fluxes: A Compilation of Absolute Stellar Fluxes Measured by the Sky Survey Telescope (S2/68) aboard the ESRO Satellite TD-1 (London: Sci. Res. Council)

Valenti, J. A., Fallon, A. A., \& Johns-Krull, C. M. 2003, ApJS, 147, 305

Wahhaj, Z., Koerner, D. W., Ressler, M. E., Werner, M. W., Backman, D. E., \& Sargent, A. I. 2003, ApJ, 584, L27

Wyatt, M. C. 2005, A\&A, 433, 1007 